\title{
Excitation of the lowest autoionizing level of caesium atoms by positron impact
}

\author{
Munywoki A. W. ${ }^{1}$, Okumu J. ${ }^{1}$, Singh C. S..$^{*}$ \\ ${ }^{1,1}{ }^{*}$ Department of Physics, Kenyatta University, P.O Box 43844-0100, Nairobi, Kenya \\ ${ }^{1 *}$ Corresponding Author. Email: singh.chadra@ku.ac.ke
}

Received: 15 July 2020 / Accepted: 15 October 2020 / Published online: 29th January 2021

\begin{abstract}
We have obtained differential and integral cross sections for the excitation of caesium atom to the lowest autoionizing level by positron impact using the distorted wave approximation. We have also done calculations for electron impact excitation of the same level using the same method and compared our present positron/electron impact results with the available theoretical positron/ electron impact results and experimental electron impact results. It is found that the present positron impact integral cross sections are higher than the cross sections of Pangantiwar and Srivastava at low and intermediate energies, but the results converge as the energy increases in the high energy range. The disagreement at near threshold energies is due to the different distortion potentials used in the two calculations. The charge on the projectile is also a determining factor in the shape of the scattering cross section curves.
\end{abstract}

Key Words: Differential cross section, integral cross section, positron impact excitation, autoionizing state, distorted wave method, atomic collision.

\section{INTRODUCTION}

An autoionizing level lies energetically above the first ionization threshold of an atom. Once excited to the autoionizing level, the atom may decay into the ground state of the atom by photon emission. The excited atoms can also decay to the ground state of the corresponding ion by electron emission. This latter process is known as autoionization. It is well known that the single ionization of heavy alkali atoms is essentially influenced by the autoionization process (Borovik \& Kupliauskiene, 2009). Previous distorted wave approximation studies on electron impact excitation of autoionizing states of alkali atoms by Pangantiwar and Srivastava (1987) reported a near threshold maximum in the total cross section results for all alkali atoms. For positrons, as will be reported later in this paper, the total cross sections trend was different at near threshold. Relativistic Distorted Wave Approximation and Relativistic Born Approximation studies on the excitation of alkalis to the lowest autoionizing levels by electron impact by Kaur and Srivastava (1999) also reported a near threshold maximum for the total cross section for all alkalis. Borovik et al. (2011) also carried out experimental studies on the excitation of caesium by electron impact and realized the same peak just above the threshold and a sharp minimum following immediately after the peak. It would thus be interesting to present our results for comparison with all these previous results, albeit with a change in the choice of atomic wave functions and the distorting potential. We also endeavor to present our results for comparison with future experimental studies on positron scattering by caesium atoms. The study of autoionizing states provides information about the atomic structure and the dynamics of a state's excitation since the cross section depends strongly on the impact energy (Theodosiou, 1987).

In this paper, we present our results for the excitation of the lowest autoionizing state of caesium by positron impact using the first order Distorted Wave Born Approximation (DWBA1) method. We also present for comparison and validation purposes, our electron impact excitation results for projectile energies between $13.5 \mathrm{eV}$ and $1000 \mathrm{eV}$. Finally, we also compare our present results with previous theoretical and experimental results. 


\section{THE THEORETICAL METHOD}

\section{The distorted wave method}

The Hamiltonian of the system is given by a combination of the atomic Hamiltonian and the Hamiltonian of the projectile as

$H=H_{a}+T+V$

where $H_{a}$ is the Hamiltonian of the isolated atom, $T=-\frac{1}{2} \nabla_{0}^{2}$ is the kinetic energy operator for the projectile and $V$ is the interaction potential between the projectile (positron) and N-electron atom given by

$V=\frac{N}{r_{0}}-\sum_{i=1}^{N} \frac{1}{r_{0 i}}$

where $\frac{N}{r}$ is the projectile-nuclear interaction, while the second term represents the projectile interaction with atomic electrons.

The total wave function $\Psi_{i}^{+}$is a solution to the Schrödinger equation

$(H-E) \Psi_{i=0}^{+}$

The superscript + indicates the usual outgoing wave boundary conditions. In this case, the projectile positron undergoes either elastic or inelastic collision with an N-electron atom. The exact T-matrix in the two potential approach is given by Madison and Bartschat (1996)

$T_{i f}=\left\langle\chi_{f}^{-}(0) \Psi_{f}(1, \ldots . N)\left|V-U_{f}\right| \Psi_{i}^{+}(0, \ldots \ldots . N\rangle\right.$

$\left\langle\chi_{f}^{-}(0) \psi_{f}(1, \ldots . . N)\left|U_{f}\right| \psi_{i}(1, \ldots . N) \phi_{i}(0)\right\rangle$

where $\psi_{i}$ and $\psi_{f}$ are properly antisymmetrized initial and final atomic wave functions for the isolated atom which diagonalize the atomic Hamiltonian according to

$\left\langle\psi_{n \prime}\left|H_{a}\right| \psi_{n}\right\rangle={ }_{\mathrm{wn}} \delta_{n n^{\prime}}$

where $w_{n}$ is the energy of the $\mathrm{n}^{\text {th }}$ state of the atom. The total wave function $\Psi_{i}^{+}$can be expressed in terms of the product of the initial distorted wave $\chi_{i}^{+}$and the initial atomic wave function $\psi_{i}$ and, using the total Green's function, it can be written as (Madison \& Bartschat, 1996)

$\Psi_{i}^{+}=\left[1+G^{+}\left(V-U_{i}\right)\right] \psi_{i} \chi_{i}^{+}$

where the full Green's function is given by

$G^{+}=(E-H+i \varepsilon)^{-1}$

and can be expanded using the distorted Green's function and an arbitrary potential $U$ as

$G^{+}=g^{+}+g^{+}(V-U) g^{+}+g^{+}(V-U) g^{+}(V-U) g^{+}+\ldots \ldots$

where the distorted Green's function is given as

$g^{+}=\left(E-h_{a}-T-U+i \epsilon\right)^{-1}$

With these expansions of the total wave function, the transition matrix can be expressed as 
$T_{i f}=T_{1}+T_{2}+\cdots$

where

$T_{1}=\left\langle\chi_{f}^{-}(0) \psi_{f}(1, . . N)\left|V-U_{f}\right| \psi_{i}(1, . . N) \chi_{i}^{+}(0)\right\rangle+\left\langle\chi_{f}^{-}(0) \psi_{f}(1, . . N)\left|U_{f}\right| \psi_{i}(1, \ldots N) \phi_{i}(0)\right\rangle$

$T_{2}=\left\langle\chi_{f}^{-}(0) \psi_{f}(1, \ldots . N)\left|\left(V-U_{f}\right) g^{+}\left(V-U_{i}\right)\right| \psi_{i}(1, \ldots . N) \chi_{i}^{+}(0)\right\rangle$

When the transition matrix is approximated as $T_{i f}=T_{1}$, it gives the first order distorted wave Born approximation. When $T_{\text {if }}$ is approximated as $T_{\text {if }}=T_{1}+T_{2}$, it gives the second order distorted wave Born approximation and so on and so forth. In this study, we consider the DWBA1 to be the transition matrix. Since the transition under consideration is from $5 p$ to 6s, all the terms with $U_{f}$ vanish due to the orthogonality of the wave functions since $U_{f}$ is a linear combination of static potentials. Again, it is important to note that the transition matrix in consideration is the direct transition matrix only since the positron and the target atom electron are distinguishable and therefore not exchangeable. Further, since the inner shell electrons in the caesium atom are attracted by the strong electrostatic force of the nucleus forming a frozen core, the caesium atom is being considered as a one-electron atom in which an electron from the $5 \mathrm{p}$ orbital is excited to the $6 \mathrm{~s}$ orbital. Therefore, the first order direct transition matrix transforms to

$T=\left\langle\chi_{f}^{-}\left(r_{0}\right) \psi_{f}\left(r_{1}\right)\left|V\left(r_{0}, r_{1}\right)\right| \psi_{i}\left(r_{1}\right) \chi_{i}^{+}\left(r_{0}\right)\right\rangle$.

The positron-target interaction term is given by

$V\left(r_{0}, r_{1}\right)=\frac{1}{r_{0}}-\frac{1}{r_{01}}$

where the radii $r_{0}$ and $r_{1}$ are the distances from the projectile positron and the atomic electron to the nucleus respectively, and $r_{01}$ is the distance between the projectile and the target atomic electron. The wave functions $\psi_{i, f}$ are the initial and final caesium atomic wave functions, and $\chi_{i, f}^{ \pm}$are the initial and final distorted waves of the projectile where \pm signifies the outgoing and incoming wave boundary conditions. The distorted waves can be expanded into partial waves as (Madison \& Bartschat, 1996)

$$
\begin{aligned}
& \left|\chi_{l_{i}}^{+}\right\rangle=\sqrt{\frac{2}{\pi} \frac{1}{k_{i} r}} \sum_{l_{i} m_{i}} i^{l_{i}} \chi_{l_{i}}\left(k_{i}, r\right) Y_{l_{i} m_{i}}(\hat{\mathrm{r}}) \gamma_{l_{i} m_{i}}^{*}\left(\hat{\mathrm{K}}_{i}\right) \\
& \left|\chi_{l_{f}}^{-}\right\rangle=\sqrt{\frac{2}{\pi}} \frac{1}{k_{f} r} \sum_{l_{f} m_{f}} i^{l_{f}} \chi_{l_{f}}\left(k_{f}, r\right) Y_{l_{f} m_{f}}(\hat{\mathrm{r}}) \gamma_{l_{f} m_{f}}^{*}\left(\dot{\mathrm{K}}_{f}\right)
\end{aligned}
$$

where the radial part of the distorted waves is a solution to the Schrödinger equation

$\left[\frac{d^{2}}{d r^{2}}-\frac{l_{s}\left(l_{s}+1\right)}{r^{2}}-U_{s}(r)+k_{s}^{2}\right] \chi_{l_{s}}^{ \pm}(r)=0$

where in $s=i, f$ and in the asymptotic region satisfies the conditions

$\lim _{r \rightarrow \infty} \chi_{l_{s}}\left(k_{s}, r\right)=j_{l_{s}}+\beta_{l_{s}}\left(-\eta_{l_{s}}+i j_{l_{s}}\right)$

where $i_{l_{s}}$ and $\eta_{l_{s}}$ are regular and irregular Ricatti-Bessel functions,

$\beta_{l_{s}}=\exp \left(i \delta_{l_{s}}\right) \sin \delta_{l_{s}}$

and $\delta_{l_{s}}$ is the elastic scattering phase shift. 
We solved (17) using Numerov's method to obtain numerical solutions which were fed to the radial mesh for solution of the transition matrix.

\section{The atomic wave functions}

The atomic wave functions used in (13) are constructed using Roothan-Hatree-Fock multi-zeta (MZ) wave functions as given in the McLean and McLean (1981) atomic data tables.

The total wave function for an N-electron atom can be given as

$\Psi=A\left(\psi_{1}^{(1)}, \psi_{2}^{(2)}, \ldots \ldots . \psi_{n}^{(n)}\right)$

where $\mathrm{A}$ is the antisymmetrizing operator and $\psi_{1}^{(1)}, \psi_{2}^{(2)}, \ldots \ldots \psi_{n}^{(n)}$ are the spin orbital one electron wave functions. The spin orbital functions are products of the spin functions and the orbital functions. These orbital functions as well as the spin functions are taken to be orthogonal to each other. The orbitals can be expanded in terms of the basis functions as

$\psi_{i \eta \gamma}=\sum_{p} \phi_{p \eta \gamma} C_{i \eta p}$

where the index $\eta$, indicates the symmetry species corresponding to the angular momentum quantum number $l$. The index $\gamma$ indicates the subspecies which label individual members of the degenerate set that transform according to the representation $\eta$. $i$ refers to the $i^{t h}$ orbital of symmetry $\eta$ which also characterizes the orbitals (McLean \& McLean, 1981). The basis functions $\phi_{p \eta \gamma}$ are Slater orbitals with both radial and angular components and are represented as

$\phi_{p \eta \gamma}(r, \theta, \varphi)=R_{\eta p}(r) \Upsilon_{\eta \gamma}(\theta, \varphi)$

where the radial part $R_{\eta p}(r)$ is given by

$R_{\eta p}(r)=\left[\left(2 \mu_{\eta p}\right) !\right]^{\frac{1}{2}}\left(2 \xi_{\eta p}\right)^{\mu_{\eta p}+\frac{1}{2}} r^{n_{\eta p}-1} e^{-\xi_{\eta p} r}$

and $\Upsilon_{\eta \gamma}(\theta, \varphi)$ are the normalized spherical harmonics in complex form.

\section{The distorting potentials}

In principle, $U_{i}$ and $U_{f}$ can be any potential as long as $\chi_{f}^{-}$and $\chi_{i}^{+}$satisfy the boundary conditions (Madison $\&$ Bartschat, 1996). Here, we choose the static potential of the target atom in its initial state as the distorting potential of the projectile in the initial channel, and a linear combination of the initial and final state static potentials of the atom to be the distorting potential in the final channel. This is because, in the initial state of the projectile, it only 'sees' the initial-state static potential of the target atom, but when the energy is transferred to the atom, it takes some time (relaxation time) before the atom goes to its final state, as a result the projectile in its final state 'sees' an intermediate potential between the initial and the final state static potentials of the target (Singh, 2004).

Therefore, the initial and final distorting potentials are given as

$U_{i}=\left\langle\psi_{i}|V| \psi_{i}\right\rangle$

$U_{f}=\frac{1}{2}\left\langle\psi_{i}|V| \psi_{i}\right\rangle+\frac{1}{2}\left\langle\psi_{f}|V| \psi_{f}\right\rangle$

\section{Total and differential cross sections}

The differential cross section is found using the standard formula (Joachain, 1975)

$\left(\frac{d \sigma}{d \Omega}\right)_{5 p \rightarrow 6 s}=\frac{1}{4 \pi^{2}} \frac{k_{f}}{k_{i}} \sum_{m=-1}^{+1}\left\{\frac{1}{4}\left|T_{5 p \rightarrow 6 s}^{D}+T_{5 p \rightarrow 6 s}^{e x}\right|^{2}+\frac{3}{4}\left|T_{5 p \rightarrow 6 s}^{D}-T_{5 p \rightarrow 6 s}^{E x}\right|^{2}\right\}$ 
Since there is no possibility of exchange between the atomic electron and the projectile positron, $T_{5 p \rightarrow 6 s}^{E x}=0$ and thus the differential cross section is given as

$\left(\frac{d \sigma}{d \Omega}\right)_{5 p \rightarrow 6 s}=\frac{1}{4 \pi^{2}} \frac{k_{f}}{k_{i}} \sum_{m=-1}^{+1}\left\{\left|T_{5 p \rightarrow 6 s}^{D}\right|^{2}\right\}$

The total cross section is obtained by

$\sigma_{\text {tot }}=\int\left(\frac{d \sigma}{d \Omega}\right)_{5 p \rightarrow 6 s} \sin \theta d \theta d \varphi$

We have modified the DWBA1 program written by Madison and Bartschat (1996) so that it can be used for the present problem of positron-caesium scattering for a $p \rightarrow s$ transition. The original program is written for electron/positronhydrogen scattering for $s \rightarrow s$ and $s \rightarrow p$ transitions.

\section{RESULTS AND DISCUSSIONS}

\section{Integral cross section}

We hereby present in Figure 1 our MZ integrated cross sections for the excitation to the lowest autoionizing state $\left(5 p^{5} 6 s^{2}\right)$ of caesium for both electron and positron impact. The present positron impact total cross sections are higher than the cross sections of Pangantiwar and Srivastava (1987) at low and intermediate energies. But as the energy increases, they converge at high energies. The disagreement at near threshold energies is due to the change of distortion potentials where, in the initial channel, Pangantiwar and Srivastava (1987) used the initial state static potential and in the final channel they used the final state static potential. In our case, as mentioned earlier, we used the initial state static potential in the initial channel and an average of the initial state static potential and excited state static potential in the final channel. The effect of the distortion potential is more at low energies compared to high energies because the time spent by the projectile in the vicinity of the target is more at low energies than at high energies.

On comparison with present electron scattering results and the electron results of Pangantiwar and Srivastava (1987), it is noted that the electron scattering total cross sections attain a sharp maximum just above the excitation threshold, a feature not observed in positron scattering results. This is due to the formation of a negative resonant ion between the projectile and the target, which decays to an autoionizing state. In the case of positron scattering, at energies near threshold, the positronium formation does not lead to an autoionizing state.

Another factor that led to the difference in scattering cross sections near the excitation threshold could be the comparative interaction of the projectile with the nucleus of the target atom. Whilst an electron experiences attraction towards the nucleus of the target once it penetrates the electron cloud, the positron undergoes repulsion from the nucleus. Thus, the low cross section values observed for positron scattering is also due to less interaction occasioned by the repulsion from the nucleus. Our electron impact distorted wave with exchange (DWE) scattering results show a higher peak near excitation threshold compared to present electron distorted wave direct (DWD) results. This is because exchange is dominant at low projectile energies. Present DWE results are higher than the DWE results of Pangantiwar and Srivastava (1987). This is due to the use of the Ochkur approximation in the DWE calculation by Pangantiwar and Srivastava (1987). We obtained the DWE results without this approximation.

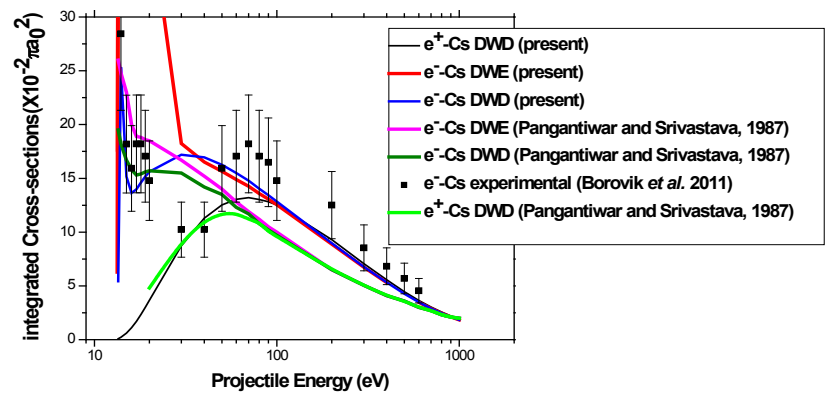

Figure 1: Total cross sections for electron and positron impact excitation of the lowest autoionizing state of caesium

Nevertheless, there was general qualitative agreement between the present positron DWD results with the positron DWD results of Pangantiwar and Srivastava (1987) and the present electron DWD and DWE results with the DWD and DWE results of Pangantiwar and Srivastava (1987).

We also compared our results with the experimental electron results of Borovik et al. (2011). The present unresolved state electron DWE results are in good qualitative agreement with the resolved ${ }^{2} \mathrm{P}_{3 / 2}$ total cross section results of Borovik et al. (2011). The possible source of quantitative differences could be due to the noninclusion of the exchange potential which caters for the exchange of the projectile electron with the target atomic 
electron; the polarization potential and the absorption potential which caters for the removal of particles from the initial channel in the calculation of the distorting potential. It also has to be noted that the Borovik et al. (2011) results provided were for only the resolved ${ }^{2} \mathrm{P}_{3 / 2}$ state, thus neglecting the contribution of the ${ }^{2} \mathrm{P}_{1 / 2}$ state which contributes significantly in the total cross section (Borovik et al., 2011).

\section{Differential Cross sections}

We also present in Figures 2-6 positron impact MZ differential cross sections for low, intermediate, and high energies and compare them with the present electron DWD and DWE results and the electron DWE results of Pangantiwar and Srivastava (1987). At energies near the excitation threshold, the graphs show a possibility of equivalent scattering to all angles. This is because at low energies the projectile loses most of its energy to the excitation process and thus it remains in the vicinity of the target for longer, allowing scattering in different directions. As the projectile energy increases, more scattering shifts to low scattering angles. This is because the projectile spends less and less time in the vicinity of the target and thus scattering is minimal. The difference in the cross sections between our present results and those of Pangantiwar and Srivastava (1987) is due to the difference in the distortion potentials used as stated earlier.

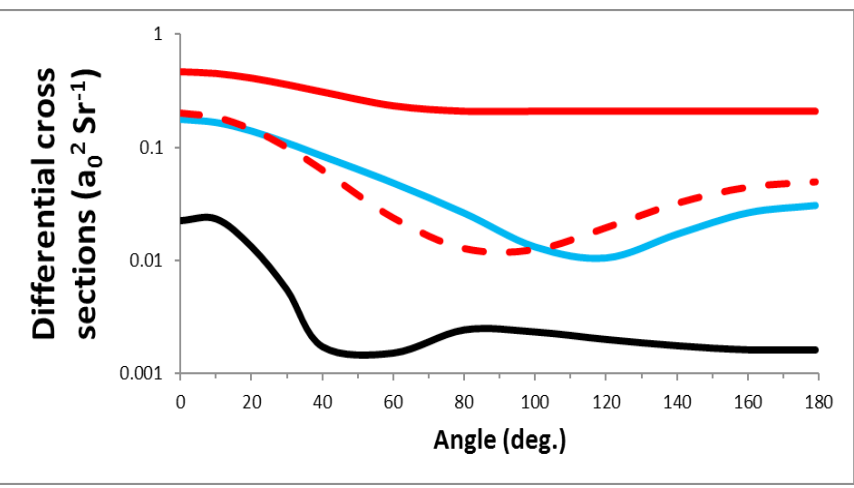

Figure 2: Differential cross sections at $16 \mathrm{eV}$ positron DWD results (present), electron DWE (present), electron DWD (present), electron DWE (Pangantiwar \& Srivastava, 1987)

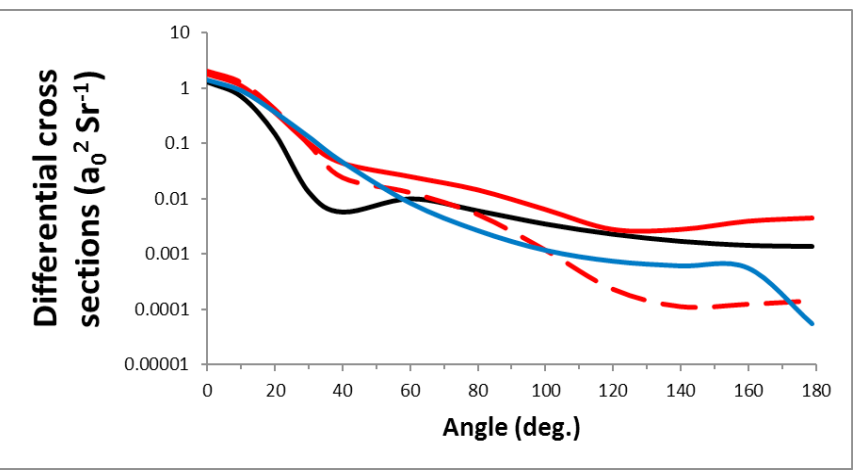

Figure 3: Differential cross sections at $30 \mathrm{eV}$ positron DWD results (present), electron DWE (present), electron DWD (present), electron DWE (Pangantiwar \& Srivastava, 1987)

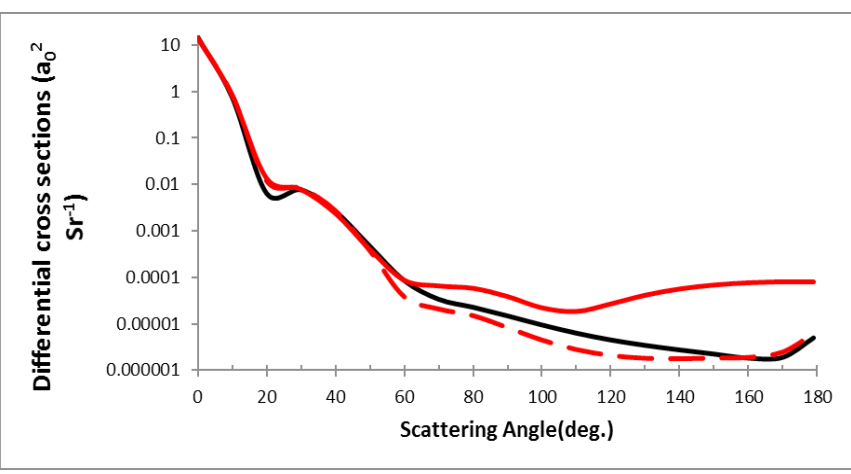

Figure 4: Differential cross sections at $100 \mathrm{eV}$ positron DWD results (present), electron DWE (present), electron DWD (present)

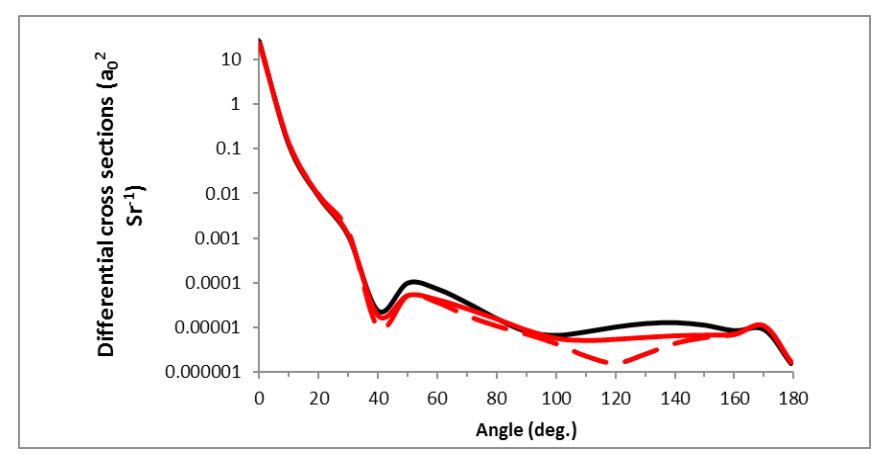

Figure 5: Differential cross sections at $200 \mathrm{eV}$ positron DWD results (present), electron DWE (present), electron DWD (present)

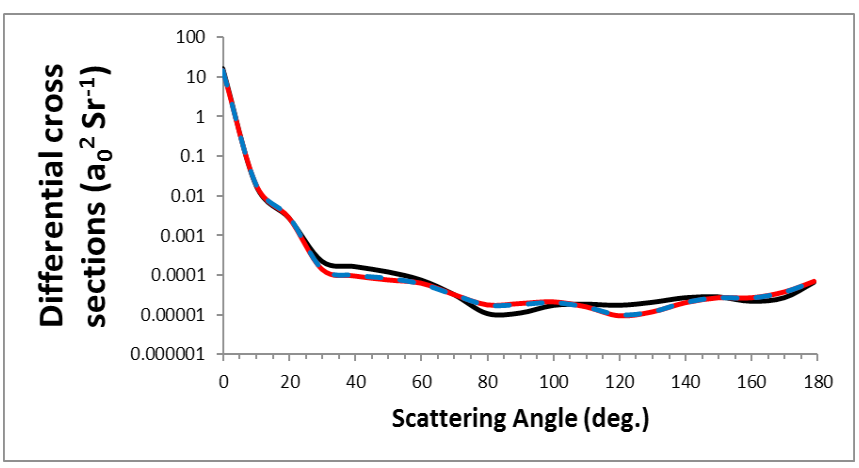

Figure 6: Differential cross sections at $500 \mathrm{eV}$ positron DWD results (present), electron DWE (present), electron DWD (present)

\section{CONCLUSION}

We have presented our calculated total and differential cross sections for positron scattering by neutral caesium atoms for energies between 13.5 and $1000 \mathrm{eV}$. Our positron and electron results are in fair agreement with the results of Pangantiwar and Srivastava (1987) except at low and intermediate energies. This difference is due to the choice of the distorting potential and the atomic wave functions. Our electron impact total cross section results are also in fair agreement with the experimental $6 s^{2},{ }^{2} \mathrm{P}_{3 / 2}$ cross sections of Borovik et al. (2011), the difference arising from their non-inclusion of the $6 \mathrm{~s}^{2},{ }^{2} \mathrm{P}_{1 / 2}$ cross sections whose contribution at low energies is more than at higher energies (Borovik et al., 2011). At intermediate energies, 
our results are within the $30 \%$ error of the Borovik et al. (2011) results. Therefore, from this study we can say that the present distorted wave method is suitable for the study of such collision processes of electron and positron impact excitation of the autoionizing states. Since no experimental results for positron scattering were available to us, we hope that our results will be useful for comparison with future experimental results.

\section{ACKNOWLEDGEMENT}

We thank the Department of Physics, Kenyatta University for providing the facility to work on this project.

\section{REFERENCES}

Borovik, A., \& Kupliauskiene, A. (2009). The $5 \mathrm{p}^{6}$ autoionisation cross section of caesium atoms: contribution to single ionization by electron impact. Journal of Physics B: Atomic, Molecular and Optical Physics, 42(16), 165202.

Borovik, A., Kupliauskiene, A., \& Zatsarinny, O. (2011). Excitation cross sections and spectroscopic classification of autoionizing levels in a caesium atom. Journal of Physics B: Atomic, Molecular and Optical Physics, 44(14), 145203.

Joachain, C. J. (1975). Quantum Collision Theory. New York: Oxford American Elsevier Publishing Company. Pp. 541.

Kaur, S., \& Srivastava, R. (1999). Excitation of the lowest autoionizing $n p^{5}(n+1) s^{2},{ }^{2} P_{3 / 2,1 / 2}$ states of $\mathrm{Na}(\mathrm{n}=2)$, $\mathrm{K}(\mathrm{n}=3), \mathrm{Rb}(\mathrm{n}=4)$ and $\mathrm{Cs}(\mathrm{n}=5)$ by electron impact. Journal of Physics B: Atomic, Molecular and Optical Physics, 32(10), 2323-2342.

Madison, D. H., \& Bartschat, K. (1996). The distortedwave method for elastic scattering and atomic excitation. In Computational Atomic Physics, pp. 6586. Springer, Berlin, Heidelberg.

McLean D.N., \& McLean R.S. (1981). RoothanHatree-Fock Atomic wave functions Slater Basis-Set Expansions for $\mathrm{Z}=55-92$. Atomic Data and Nuclear Data tables, 26, 197-387.

Pangantiwar, A., \& Srivastava, R. (1987). $e^{ \pm}$impact excitation of autoionizing levels in alkalis: a distorted wave approach. Journal of Physics B: Atomic, Molecular and Optical Physics, 20(21), 5881-5902.

Singh, C. S. (2004). Magnetic-sublevel differential cross sections for electron impact excitation of $2^{1} \mathrm{P}$ state of helium. East African Journal of Physical Sciences, 5, 85-98.

Theodosiou, C. E. (1987). Collisional excitation and alignment of $\mathrm{np}^{5}(\mathrm{n}+1) \mathrm{s}^{2}$ autoionizing states of the alkali-metal atoms. Physical Review A, 36(7), 31383144 . 